\title{
Node Localization Algorithm of Non-line-of-sight Environment Based on Particle Swarm Optimization
}

\author{
Zhang $\mathrm{Hao}^{1}$ \\ ${ }^{1}$ Inner Mongolia Normal University, HOHHOT Inner Mongolia, 010020, China \\ E-mail: zhanghao8356@163.com
}

\begin{abstract}
Node localization algorithm of NLOS (Non-line-of-sight) environment based on PSO (particle swarm optimization) is proposed aiming at NLOS range error. PSO algorithm is quoted in wireless sensor network localization. First of all, the parameter of PSO algorithm is improved and nonlinear adjustment to inertia weight is made to boost convergence rate of algorithm, at the same time, target value is in rank ordering to decrease calculated quantity. Simulation results demonstrate that proposed algorithm reduced error influence of NLOS and improved location accuracy.
\end{abstract}

Keywords: Wireless sensor networks; Node location; Optimization algorithm; Weight; Adjustment

\section{Introduction}

A series of nodes required to be installed in environment to shape WSNs (Wireless Sensor Networks) [1] so as to get special tasks done. Each node will be at least installed one wireless transceiver, micro-processor and a series of sensors to be used for detecting neighboring mode and measuring reciprocal distance. In addition, there exist special node of additional functions such as mobile node and GPS (Global Position systems). For WSNs applications such as environmental remote sensing, structural monitoring and mobile target tracking, localization has become a key technology. It's significantly critical to design effective location algorithm in WSNs for the reason that most of applications rest upon accurate positioning. The accuracy of ranging has a direct influence on ranging localization algorithm. However, NLOS will make an impact on wireless environment ranging. A large number of research and analysis show that NLOS error is the major error source of range-based localization. Location algorithm based on maximum posterior probability estimation is proposed in literature [6]. For one thing, maximum posterior probability estimation is used to make NLOS detection for range error, then location result with significant error will be abandoned, at last, weighted least squares is used to locate. Although such algorithm is provided with higher location accuracy, the detection to NLOS error is quite complex and power dissipation is bigger. Chen et al. [7] Put forward a kind of location algorithm that help lower the influence what NLOS error brings about. For one thing, estimate residual error of ranging in each group with least square method, then weight on location result according to the size of residual error in order to reduce the influence of NLOS error on location accuracy. Li et al. [8] Put forward IMR algorithm of residual error location with iterative least. Such algorithm first restrict the largest number of iteration. Then estimate the residual error ranging in each group, at last, position estimation will be carried out on the group with minimum residual error of ranging, such algorithm however is not provided with high location accuracy.

Such algorithm above are attached with issues of complexity or worse location accuracy, therefore, researchers exert themselves to be in search of position 
algorithm with low resources consuming and high position accuracy. PSO (Particle Swarm Optimization) algorithm is a fine choice. For this reason, this article employs PSO algorithm to wireless sensor network node localization of NLOS environment and put forward mode localization algorithm of NLOS environment based on PSO, inertia weight based on particle swarm is improved with such algorithm so as to lower the influence of NLOS on location accuracy and, in the meantime, to boost convergence rate of algorithm.

\section{Constraint Condition and Problem Description}

\subsection{System Model}

Consider an wireless locating network of $M$ unknown node and $N$ anchor node whose are distributed in two dimensional spaces. Let $v_{a}=\{1,2, \cdots, M\}$ and $v_{A}=\{M+1, M+2, \cdots, M+N\}$ are unknown node set and anchor node set, respectively. Position identification of unknown node $i \in v_{a}$ is $\mathrm{p}_{i}=\left(x_{i}, y_{i}\right)$. Accordingly, position vector of $M$ unknown node is $\mathbf{p}=\left[\mathrm{p}_{1}^{T} \mathrm{p}_{2}^{T} \cdots \mathrm{p}_{M}^{T}\right]$. Position identification of anchor node $j \in v_{A}$ is $L_{i}=\left(x_{i}^{\prime}, y_{i}^{\prime}\right)$.

For each pair of nodes, consider ranging value of $R$ group to carry out distance estimation. True range of node $i$ and node $j$ is $d_{i j}$. The $n$th ranging value to distance value $\hat{d}_{i j}[n]$ of $d_{i j}$ :

$$
\hat{d}_{i j}[n]=\left\|\mathrm{p}_{i}-\mathrm{p}_{j}\right\|+\mathbf{z}_{i j}[n]+\mathbf{b}_{i j}[n], n=1,2, \cdots, R
$$

where, $\mathbf{z}_{i j}[n]$ is Gauss measurement noise with zero mean value, and $\mathbf{z}_{i j}[n] \sim \mathbf{N}\left(0, \sigma_{i j}^{2}\right)$. $\mathbf{b}_{i j}[n]$ is ranging error results from NLOS, and mean value $E\left\{b_{i j}[n]\right\}=\mu_{i j}$, variance $\operatorname{Var}\left\{b_{i j}[n]\right\}=\zeta_{i j}^{2}$.

To solve LS location problem, sample mean of $R$ th ranging value is quoted:

$$
\hat{d}_{i j}=\frac{1}{R} \sum_{n=1}^{R} \hat{d}_{i j}[n]=\left\|\mathrm{p}_{i}-\mathrm{p}_{j}\right\|+\mathbf{z}_{i j}+\mathbf{b}_{i j}
$$

Where, $\mathbf{z}_{i j}=(1 / R) \sum_{n=1}^{R} z_{i j}[n], \mathbf{b}_{i j}=(1 / R) \sum_{n=1}^{R} b_{i j}[n]$.

Deviations of variable $b_{i j}[n]$ of NLOS is random variable of independent identically distributed, estimate the variance of $b_{i j}[n]$ with sample variance of free bias of $\hat{d}_{i j}[n]$ :

$$
\hat{\zeta}_{i j}^{2}=\frac{1}{R-1} \sum_{n=1}^{N}\left(\hat{d}_{i j}[n]-\hat{d}_{i j}\right)^{2}-\sigma_{i j}^{2}
$$

\subsection{Problem description}

This section explains the influence of ranging error on localization as an example of least square method. Let $\mathbf{x}_{i}$ represent unknown node location of $i$, and $\mathrm{x}=\left[\mathrm{x}_{1}^{T} \mathrm{x}_{2}^{T} \cdots \mathrm{x}_{M}^{T}\right]$. Location of LS cooperative localization of position vector estimates $\mathrm{P}$ as:

$$
\hat{\mathrm{p}}=\underset{\mathrm{x} \in \mathrm{R}^{2}}{\operatorname{argmin}} f_{\mathrm{LS}}(\mathrm{x})
$$

where:

$$
f_{\mathrm{LS}}(\mathrm{x})=\sum_{i \in v_{i} j \epsilon_{v_{i}} v_{v_{i}}} \frac{c_{i j}}{\sigma_{i j}^{2}+\zeta_{i j}^{2}}\left(\hat{d}_{i j}-\hat{b}_{i j}-\left\|\mathrm{x}_{i}-\mathrm{x}_{j}\right\|\right)^{2}
$$

Where, $\hat{b}_{i j}$ is biased estimation and $c_{i j}$ is connected factor. If node $i$ and $j$ is able to be connected with one another, $c_{i j}=1$, otherwise $c_{i j}=0$. In addition, if it's in LOS environment, $\hat{b}_{i j}$ is unbiassed estimate and $E\left\{\hat{b}_{i j}\right\}=\mu_{i j}$. The literature [11-12] put forward many a scheme on how to alleviate deviation influence of NLOS ranging.

Formula (4) and (5) show that deviations of variable $b_{i j}[n]$ of NLOS has a direct influence on $\mathrm{p}$ estimation, however, in real environment, the ranging error of NLOS is inevitable. Therefore, an ranging error of NLOS must be taken into consideration 
while location algorithm is being carried out in NLOS environment. For this reason, this article put forward NLOS+PSO of WSN to NLOS based on particle swarm optimization to alleviate the influence of NLOS ranging error on position estimation so as to boost location accuracy.

\section{NLOS+PSO Algorithm}

\subsection{Theory of PSO Algorithm}

For one thing, PSO algorithm will randomly select a set of position coordinates in a certain area and make it as initial particle. Then substitute initial particle into objective function to calculate fitness. An optimal position of particle and current optimal position of entire species will be obtained in the end. Suppose the position coordinates of $i$ th particle is $\mathbf{x}_{i}=\left(x_{i 1}, x_{i 2}, \cdots, x_{i r}\right)$, where $r$ represents spatial dimension. $v_{i}=\left(v_{i l}, v_{i 2}, \cdots, v_{i r}\right)$ is particle velocity. Each iteration will be updated according to formula (6):

$$
\begin{gathered}
v_{i}(k+1)=\omega v_{i}(k)+\beta_{1} \text { rand } 1\left(\text { pbest }_{i}-\mathbf{x}_{i}(k)\right)-\beta_{2} \text { rand }\left(\text { gbest }_{3}-\mathbf{x}_{i}(k)\right) \\
x_{i}(k+1)=x_{i}(k)+v_{i}(k+1)
\end{gathered}
$$

Where, $k$ is iteration times, $\beta_{1}$ and $\beta_{2}$ are acceleration factors, rand 1 and rand 2 are two random numbers from 0 to 1 , respectively. $\omega$ is inertia weight coefficient. pbest $_{i}$ is denoted as personal best particle that the $i$ th particle experiences, gbest $t_{g}$ is denoted as global optimal position of all particles in current group. Then to define the fitness, which is denoted as average error value of distance and ranging result between particle and each anchor node, described as formula (8).

$$
f(x, y)=\frac{1}{N} \sum_{i=1}^{M}\left(\left|\sqrt{\left(x-x_{i}\right)^{2}+\left(x-y_{i}\right)^{2}}-d_{i}\right|\right)
$$

Where, $d_{i}$ is denoted as ranging value of particle from anchor node ${ }^{i}$. Fitness is an critical parameter of PSO algorithm that will have a direct influence on location accuracy.

\subsection{Improvements of PSO Algorithm}

An optimization is carried out on inertia weight coefficient with NLOS+PSO algorithm to boost location accuracy of PSO algorithm. The greater the inertia weight coefficient is, the faster particle velocity will be, and the stronger global searching ability will be, oppositely, the stronger local search ability will be. For this reason, inertia weight coefficient will be defined according to formula (9):

$$
\omega(k)=\left(\omega_{\max }-\omega_{\min }\right) \exp \left(-\frac{k^{2}}{\left(h \cdot \text { iter }_{\max }\right)^{2}}\right)+\omega_{\min }
$$

Where, $h$ is denoted as extension constant coefficient and $k$ is denoted as current iteration times. $\omega_{\max }$ and $\omega_{\min }$ are denoted as maximum value and minimum value of inertia weight coefficient. iter $_{\max }$ is denoted as maximum iterations.

With an adjustment to value of extension constant coefficient is able to alter nonlinearity of inertia weight function. The research shows that when $h=0.2$, PSO algorithm is provided with the fastest convergence rate. Figure 1 shows how inertia weight function changes along with iteration times when $h=0.1,0.2$ and 0.3 . 


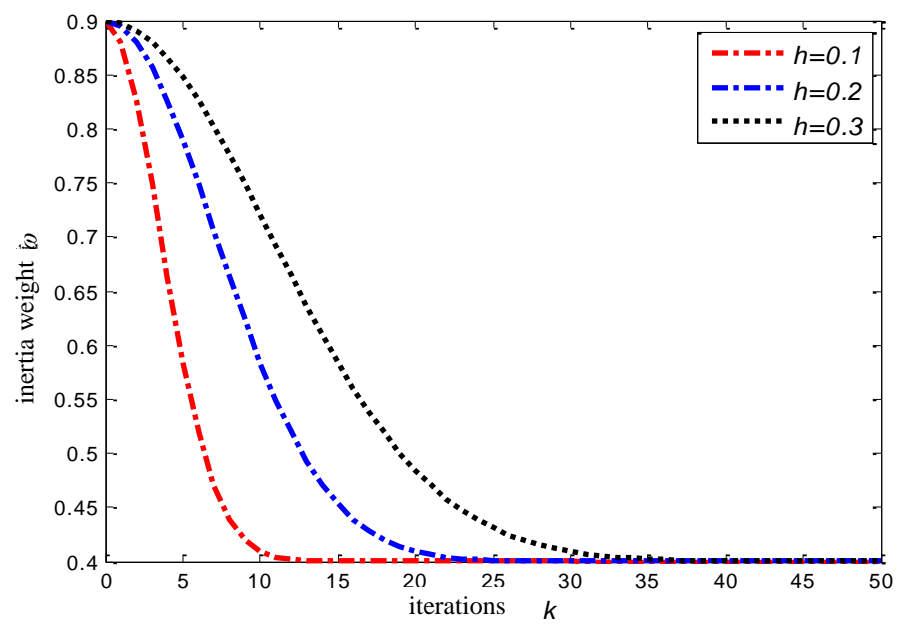

Figure 1. Inertia Weight Function

It's observed that that inertia weight function is rapidly reduced along with iteration times, as shown in Figure 1. Inertia weight function is is decreased progressively as convex function, which is in favor of globally optimal solution, at the initial stage of iteration. As the increase of iteration times, inertia weight function is decreased progressively as concave function, which is beneficial to convergence of algorithm. The whole variation curve approaches recession curve of concave function, which is identical to the conclusion of literature [13]. Under such function, an optimal positioning performance will be gained if make an adjustment to inertia weight.

In addition, convergence rate is also an important performance of PSO algorithm, therefore, to improve convergence rate, a good-and-bad ranking is required to velocity and position of particle, half of particles with poor performance shall be thrown away with each iteration and the remaining half of them with good performance will pass into next iteration.

$$
s\left(f_{\text {sorr }}(1: N)\right)=\left\{\begin{array}{l}
x_{\text {sort }}=\left(\frac{N}{2}: N\right)=x_{\text {sor }}\left(1: \frac{N}{2}\right) \\
v_{\text {sort }}=\left(\frac{N}{2}: N\right)=v_{\text {sor }}\left(1: \frac{N}{2}\right)
\end{array}\right.
$$

Where, $s\left(f_{\text {sorn }}(1: N)\right)$ is selection function, $x_{\text {sort }}$ and $v_{\text {sor }}$ are particle position and velocity position after the ranking.

\subsection{Algorithm Procedure of NLOS+PSO}

Pseudocode of NLOS+PSO algorithm is proposed according to analysis above section, as follows: 


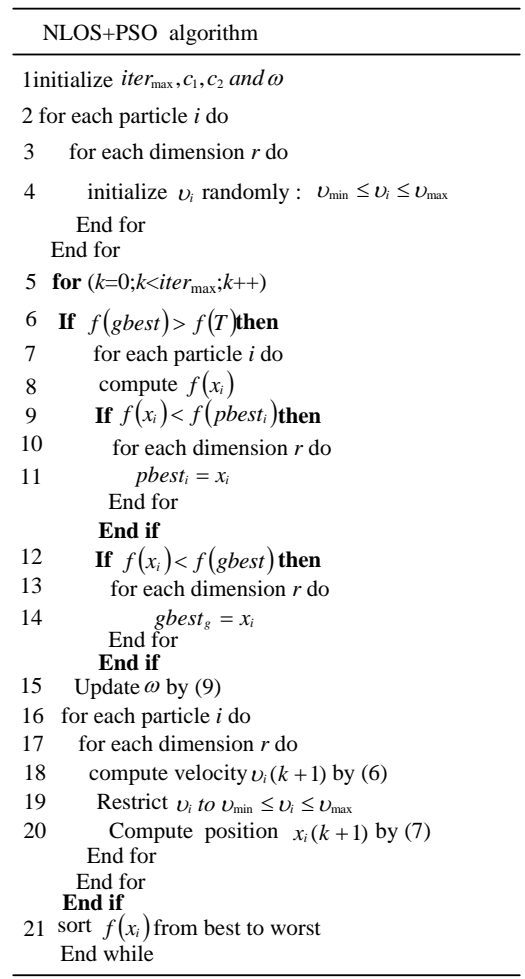

Figure 2. Pseudocode of NLOS+PSO Algorithm

\section{System Simulation}

\subsection{System Parameter}

With Metlab to establish simulation system and analyze algorithm performance. 80 sensing nodes are distributed in an area of $100 \mathrm{~m} \times 100 \mathrm{~m}$. Among it the number of anchor node changes from 5 to 30 , of which anchor node percent $p \%$ with NLOS error is provided. Communication range of node $\gamma=30 \mathrm{~m}$. acceleration coefficient $\beta_{1}$

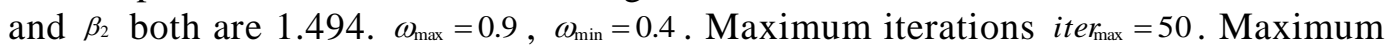
velocity of particle $v_{\max }=10$. Each experiment is repeated for 100 times and take an average value as final simulation data.

In addition, select average localization error ${ }^{e_{\text {arg }}}$ as performance index, described as formula (11).

$$
e_{\text {avg }}=\frac{\sum_{i=1}^{m} \sqrt{\left(x_{i}-\hat{x}_{i}\right)^{2}+\left(y_{i}-\hat{y}_{i}\right)^{2}}}{m \gamma}
$$

Where, $x_{i}$ and ${ }^{y_{i}}$ are denoted as real position of the ${ }^{i}$ th node. $\hat{x}_{i}$ and $\hat{y}_{i}$ both are estimation position. ${ }^{m}$ is denoted as unknown node number positioned.

\subsection{Simulation Result}

LS, IMR and traditional PSO algorithm are selected to be simulation comparisons in order to more fully analyze algorithm performance, simulation result is shown as Figure 3-4.

4.2.1. Average Localization Error: Figure 3 shows that variation curve of average positioning error along with NLOS error when 10 anchor nodes $p \%=30 \%$. It's observed that all average positioning errors of algorithm will be increased as the increase of NLOS error, as shown in Figure 3. Among it LS algorithm is provided 
with a maximum fluctuation, average positioning error of LS algorithm is up to $65 \%$ when NLOS error is reaching $7 \mathrm{~m}$. However, variation of average positioning error for IMR, PSO and NLOS+PSO algorithms, of which the average positioning error of NLOS+PSO algorithm being the least, which improves location accuracy in NLOS environment, are rather equalized.

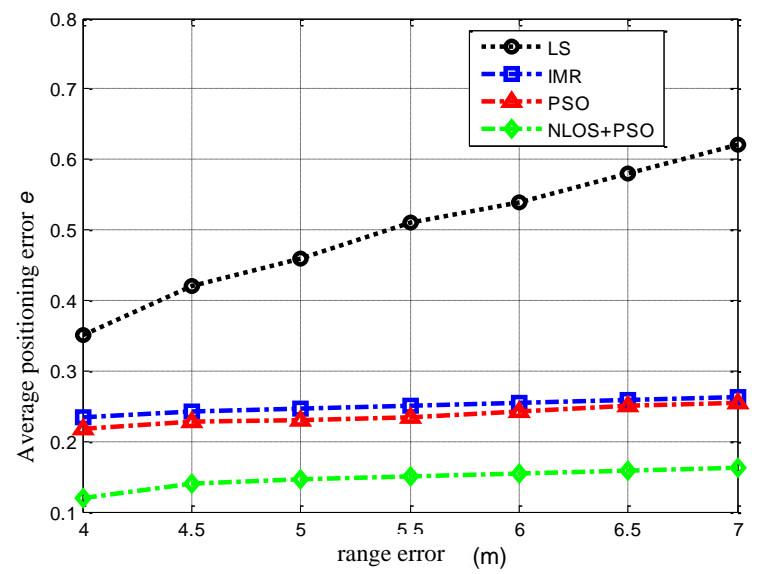

Figure 3. Variation Curve of Average Positioning Error Along with NLOS Error

Figure 4 shows how average positioning error changes along with anchor node number in Gaussian distribution environment when NLOS error is obeying mean value 6 and standard deviation 1. It's observed that average positioning error has a downtrend as the increase of anchor node, as shown in Figure 4. NLOS+PSO algorithm proposed however is provided with a rather high location accuracy, the reason is that range constraint is carried out on NLOS+PSO algorithm, with more anchor nodes come with accurate range estimation of unknown node.

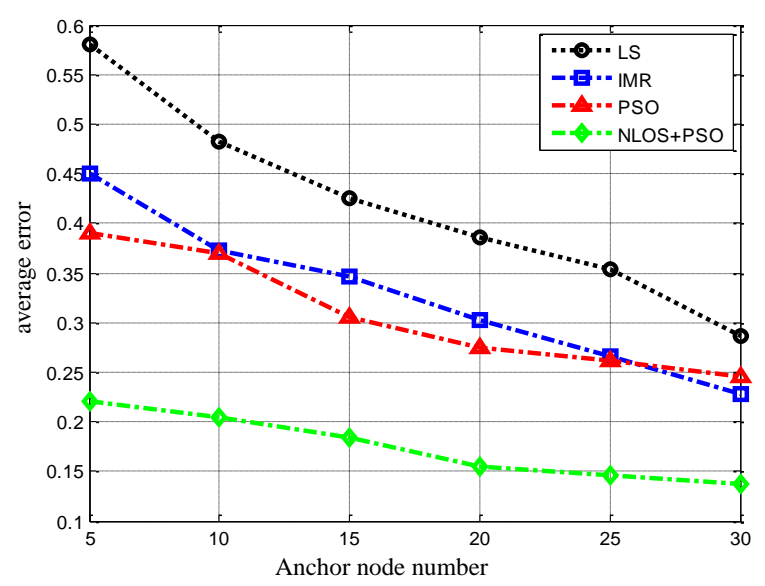

Figure 4. Variation Curve of Average Positioning Error Changes along with Anchor Node Number

4.2.2. Iterations: Iterations is an important performance of calculation algorithm, for this reason, iterations performance of traditional PSO and proposed NLOS+PSO is analyzed, the simulation is shows as Figure 5-6. 
Figure 5 (a) is uniform distribution of NLOS that obeys $4 \mathrm{~m}$ to $8 \mathrm{~m}$, the number of anchor node is iterations under a condition of 10, 15, 20 and 25, respectively. 5 (b) describes that the number of anchor node is 15 , NLOS error is iterations of algorithm under a condition of $4 \mathrm{~m}, 6 \mathrm{~m}$ and $8 \mathrm{~m}$, respectively.
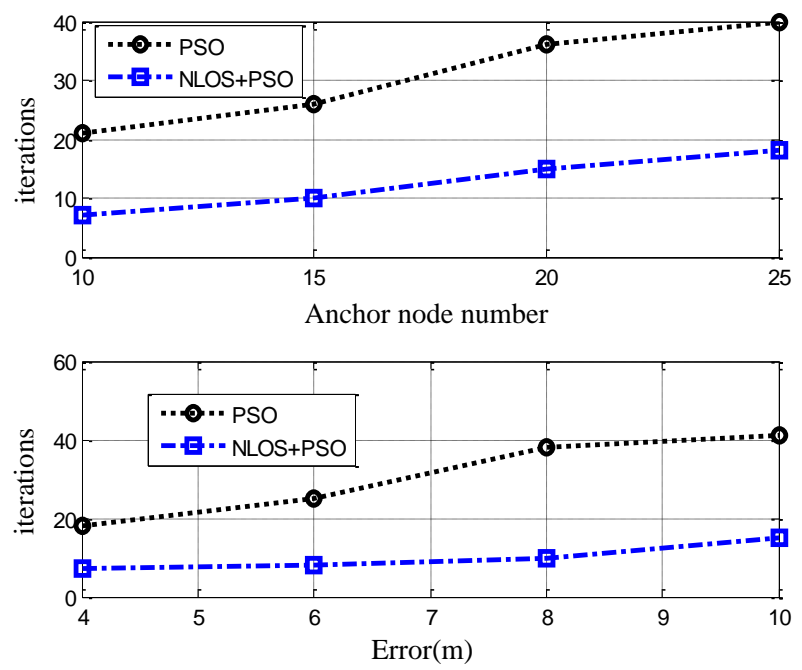

Figure 5. Iterations

It's observed that the iterations of proposed NLOS+PSO algorithm is far less than PSO, as shown in Figure 5. It lies in updating inertia weight to speed up convergence of algorithm.

4.2.3. Time: Table 1 shows time-consuming of identical iterations for each algorithm. It's observed that time-consuming of LS algorithm being the shortest, and the time-consuming of NLOS+PSO algorithm is much more than PSO, as shown in table 1. This is because NLOS+PSO algorithm is more complex in form of inertia weight, which increases calculated quantity.

Table 1. Time-consuming of Algorithm

\begin{tabular}{c|c|c|c|c|}
\hline \multirow{2}{*}{ time } & \multicolumn{4}{|c|}{ algorithm } \\
\cline { 2 - 5 } iteration & LS & IMR & PSO & NLOS+PSO \\
\hline 1 & 39.82 & 31.82 & 23.82 & 30.14 \\
\hline 10 & 41.19 & 29.23 & 30.92 & 34.02 \\
\hline 100 & 93.36 & 52.23 & 40.18 & 42.38 \\
\hline
\end{tabular}

\section{Conclusion}

NLOS environment of wireless sensor networks is a key factor to influence accuracy of ranged-based localization. For this reason, Node localization algorithm of NLOS (Non-line-of-sight) environment based on PSO (particle swarm optimization) is proposed. Nonlinear adjustment strategy of inertia weight is carried out on proposed algorithm, which helps boost convergence rate of algorithm, in the meantime, a ranking for target value is adopted to throw away particles with poor performance and decrease calculated quantity. Experimental results demonstrate that proposed NLOS+PSO algorithm improved location accuracy, restrict NLOS range error and boosted convergence rate in NLOS environment. 


\section{References}

[1] J. $\mathrm{Hu}$ and Z. Gao, "Distinction immune genes of hepatitis-induced hepatocellular carcinoma", Bioinformatics, vol. 28, no. 24, (2012), pp. 3191-3194.

[2] T. Su, W. Wang and Z. Lv, "Rapid Delaunay triangulation for randomly distributed point cloud data using adaptive Hilbert curve", Computers \& Graphics, vol. 54, (2016), pp. 65-74.

[3] W. Gu, Z. Lv and M. Hao, "Change detection method for remote sensing images based on an improved Markov random field", Multimedia Tools and Applications, (2015), pp. 1-16.

[4] Z. Chen, W. Huang and Z. Lv, "Towards a face recognition method based on uncorrelated discriminant sparse preserving projection", Multimedia Tools and Applications, (2015), pp. 1-15.

[5] D. Jiang, X. Ying and Y. Han, "Collaborative multi-hop routing in cognitive wireless networks", Wireless Personal Communications, (2015), pp. 1-23.

[6] J. Yang, S. He and Y. Lin, "Multimedia cloud transmission and storage system based on internet of things", Multimedia Tools and Applications, (2015).

[7] C. Guo, X. Liu and M. Jin, "The research on optimization of auto supply chain network robust model under macroeconomic fluctuations", Chaos, Solitons \& Fractals, (2015).

[8] X. Li, Z. Lv and J. Hu, "XEarth: A 3D GIS Platform for managing massive city information", Computational Intelligence and Virtual Environments for Measurement Systems and Applications (CIVEMSA), 2015 IEEE International Conference on IEEE, (2015), pp. 1-6.

[9] J. Yang, B. Chen and J. Zhou, "A Low-Power and Portable Biomedical Device for Respiratory Monitoring with a Stable Power Source", Sensors, vol. 15, no. 8, (2015), pp. 19618-19632.

[10] G. Bao, L. Mi, Y. Geng and K. Pahlavan, "A computer vision based speed estimation technique for localizing the wireless capsule endoscope inside small intestine", 36th Annual International Conference of the IEEE Engineering in Medicine and Biology Society (EMBC), (2014).

[11] X. Song and Y. Geng, "Distributed community detection optimization algorithm for complex networks", Journal of Networks, vol. 9, no. 10, (2014), pp. 2758-2765.

[12] D. Jiang, X. Ying and Y. Han, "Collaborative multi-hop routing in cognitive wireless networks", Wireless Personal Communications, (2015), pp. 1-23.

[13] J. Hu and Z. Gao, "Modules identification in gene positive networks of hepatocellular carcinoma using Pearson agglomerative method and Pearson cohesion coupling modularity", Journal of Applied Mathematics, (2012).

[14] D. Jiang, Z. Xu and Z. Chen, "Joint time-frequency sparse estimation of large-scale network traffic", Computer Networks, vol. 55, no. 15, (2011), pp. 3533-3547.

[15] J. Hu, Z. Gao and W. Pan, "Multiangle Social Network Recommendation Algorithms and Similarity Network Evaluation", Journal of Applied Mathematics, (2013).

[16] Y. Geng, J. Chen, R. Fu, G. Bao and K. Pahlavan, "Enlighten wearable physiological monitoring systems: On-body rf characteristics based human motion classification using a support vector machine", IEEE transactions on mobile computing, vol. 1, no. 1, (2015), pp. 1-15.

[17] Z. Lv, A. Halawani and S. Feng, "Multimodal hand and foot gesture interaction for handheld devices", ACM Transactions on Multimedia Computing, Communications, and Applications (TOMM), vol. 11, no. 1 s, (2014), p. 10.

[18] G. Liu, Y. Geng and K. Pahlavan, "Effects of calibration RFID tags on performance of inertial navigation in indoor environment", 2015 International Conference on Computing, Networking and Communications (ICNC), (2015).

[19] J. He, Y. Geng, Y. Wan, S. Li and K. Pahlavan, "A cyber physical test-bed for virtualization of RF access environment for body sensor network", IEEE Sensor Journal, vol. 13, no. 10, (2013), pp. 38263836.

[20] W. Huang and Y. Geng, "Identification Method of Attack Path Based on Immune Intrusion Detection", Journal of Networks, vol. 9, no. 4, (2014), pp. 964-971.

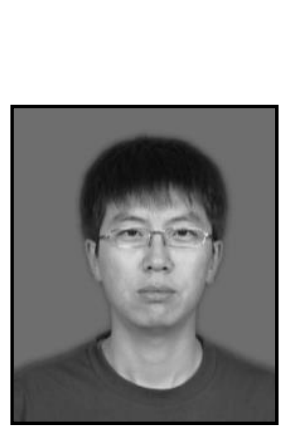

\section{Author}

Zhang Hao, he received his M.S. degree in computer education from Inner Mongolia Normal University, China. He is currently a lecturer in the International Exchange College at Inner Mongolia Normal University. He research interest is mainly in the area of Computer Software. He has published several research papers in scholarly journals in the above research areas and has participated in several books. 